\title{
Increasing delivery of an outdoor journey intervention to people with stroke: A feasibility study involving five community rehabilitation teams
}

Annie McCluskey ${ }^{1,2^{*}+}$, Sandy Middleton ${ }^{3,4 \dagger}$

\begin{abstract}
Background: Contrary to recommendations in a national clinical guideline, baseline audits from five communitybased stroke rehabilitation teams demonstrated an evidence-practice gap; only $17 \%$ of eligible people with stroke were receiving targeted rehabilitation by occupational therapists and physiotherapists to increase outdoor journeys. The primary aim of this feasibility study was to design, test, and evaluate the impact of an implementation program intended to change the behaviour of community rehabilitation teams. A secondary aim was to measure the impact of this change on client outcomes.

Methods: A before-and-after study design was used. The primary data collection method was a medical record audit. Five community rehabilitation teams and a total of 12 professionals were recruited, including occupational therapists, physiotherapists, and a therapy assistant. A medical record audit was conducted twice over 12 months (total of 77 records pre-intervention, 53 records post-intervention) against a guideline recommendation about delivering outdoor journey sessions to people with stroke. A behavioural intervention (the 'Out-and-About Implementation Program') was used to help change team practice. Active components of the intervention included feedback about the audit, barrier identification, and tailored education to target known barriers. The primary outcome measure was the proportion of medical records containing evidence of multiple outdoor journey sessions. Other outcomes of interest included the proportion of medical records that contained evidence of screening for outdoor journeys and driving by team members, and changes in patient outcomes. A small sample of community-dwelling people with stroke $(n=23)$ provided pre-post outcome data over three months. Data were analysed using descriptive statistics and t-tests.
\end{abstract}

Results: Medical record audits found that teams were delivering six or more outdoor journeys to $17 \%$ of people with stroke pre-intervention, rising to $32 \%$ by 12 months post-intervention. This change represents a modest increase in practice behaviour (15\%) across teams. More people with stroke (57\%) reported getting out of the house as often as they wanted after receiving the outdoor journey intervention compared to 35\% one year earlier; other quality of life outcomes also improved.

Conclusions: The 'Out-and-About Implementation Program' helped rehabilitation teams to change their practice, implement evidence, and improve client outcomes. This behavioural intervention requires more rigorous evaluation using a cluster randomised trial design.

\footnotetext{
* Correspondence: annie.mccluskey@sydney.edu.au

† Contributed equally

${ }^{1}$ Community-Based Health Care Research Unit, Faculty of Health Sciences,

The University of Sydney, New South Wales, Australia
} 


\section{Background}

Over 60,000 Australians experience a stroke each year [1]. Less than $10 \%$ of people with stroke can walk fast enough to cross a road safely when they leave hospital [2]. Up to $50 \%$ fall at home in the first six months after discharge [3]. Two-thirds of people are never able to resume driving after a stroke $[4,5]$, and many cannot use public transport. Unless community rehabilitation is provided, many people with stroke will experience social isolation, reduced physical activity, and poor quality of life.

\section{Evidence-based community stroke rehabilitation}

Community rehabilitation, including mobility and transport training, can improve health outcomes for people with stroke [6-8]. A systematic review of 21 trials of physiotherapy exercise programs for people with stroke reported gains in walking speed and distance following task-specific training [6]. One of these trials reported increased walking capacity following four weeks of treadmill training and overground walking practice in community-dwelling stroke survivors with speed gains being maintained after three months [8].

Yet, people with stroke who received several weeks of community mobility training report a lack of confidence negotiating ramps, escalators, and shopping malls [9]. Further, repeated practice walking indoors in a hospital gym did not automatically lead to improved walking outdoors. To gain confidence and skills, people with stroke seem to need multiple escorted journeys in their local community with a rehabilitation therapist.

Increased outdoor journeys and quality of life poststroke were the focus of one trial conducted in England [7]. This trial compared the distribution of leaflets describing local transport options (control group), with the same leaflets plus delivery of up to seven individual sessions over a three-month period by occupational therapists who encouraged outdoor mobility and travel (intervention group). Participants in the intervention group were escorted by therapists on walks, bus, and taxi trips until they felt confident to go out alone [10]. Therapists also provided transport information to the intervention group. After four months and a median of six sessions, twice as many people from the intervention group reported getting out as often as they wanted (RR 1.72 , 95\% CI 1.25 to 3.27) [7]. Between-group differences were maintained at 10 months, long after therapy had ceased.

\section{The evidence-practice gap}

Australian national stroke guidelines recommend escorted journeys, written transport information, and ambulation training following stroke [11]. These recommendations are consistent with findings from the randomised trial by Logan and colleagues $[7,10]$. However, anecdotally, a large evidence-practice gap appeared to exist in local community stroke rehabilitation practice in our region.

Barriers to translating evidence into practice include lack of knowledge about the evidence, limited skills and competence, and consumer expectations about therapy $[12,13]$. Implementation programs use a number of 'interventions' to target local barriers and change practice $[14,15]$. These interventions include dissemination of clinical guidelines and other educational materials [16], education meetings, audit and performance feedback [17], reminder systems, and a combination of these. The efficacy of implementation interventions was evaluated in a systematic review that included 235 studies $[18,19]$; in that review, most interventions led to small changes in practice of up to $10 \%$. Larger changes can be expected when compliance with best practice is low at baseline. We used this 'evidence about getting evidence into practice' to design and test an implementation program.

The primary aim of the present study was to design, pilot test, and evaluate the impact of an implementation program intended to change the behaviour of community rehabilitation teams. The behaviour measured was delivery of multiple outdoor journey sessions to people with stroke, consistent with a national guideline recommendation. A secondary aim was to evaluate the impact of practice change on client outcomes.

\section{Methods}

A before-and-after design was used. The primary data collection method was medical record audit, conducted on two cohorts: a pre-intervention cohort, and another different cohort 12 months later. A secondary data collection method was administration of standardised outcome measures to people with stroke who received the outdoor journey intervention.

\section{The Sample}

\section{Rehabilitation team participants}

A purposive sample of five community rehabilitation teams was recruited in Sydney, Australia representing different models of service delivery (out-patient, domiciliary, and day hospital). To be eligible, teams had to employ at least one occupational therapist and one physiotherapist, and have seen at least ten people with stroke in the previous six months. These professionals helped conduct medical records audits, received feedback from the audits, were interviewed about barriers to implementation, attended an education session, and delivered the outdoor journey sessions to people with stroke on their caseload. 


\section{Participants with stroke}

Therapists from two teams consented to their clients being recruited. Funding did not permit data collection across all five teams. Community-dwelling people with stroke seen by two participating teams were invited to participate in the study if they met the following criteria: they needed rehabilitation to increase their outdoor journeys (based on screening questions asked by a team member); they agreed to participate in multiple outdoor journey sessions; and they agreed to be interviewed by AM and provide additional outcome data.

\section{The out-and-about implementation program}

The intervention provided to help rehabilitation therapists implement the outdoor journeys was named the 'Out-and-About Implementation Program'. The program aimed to change practice and included three active components: medical record audits followed by feedback, barrier identification, and education to target known local barriers.

Medical record audits were conducted retrospectively by AM and two professionals from each team. We requested 100 consecutive records (20 records for each of the five teams) of people with stroke who had received therapy (for any reason) in the previous 12 months from a team occupational therapist, physiotherapist, or both. One exception was a new team that had been established six months earlier, and had only seen 10 people with stroke. In that case, we requested all of their records for people with stroke seen since service commencement. Multiple auditors were used to raise professionals' awareness of their practice, and the practice of their team, by engaging them in audits. Each professional audited at least three medical records. Two medical files from the total sample were double coded by the first investigator to check for consistency. Differences were discussed and consensus reached when necessary. No formal study of rater agreement was conducted.

Audit criteria were rated using yes/no response options. Questions were asked about screening and assessments conducted, intervention provided, goals set and outcomes measured in relation to transport, outdoor mobility, and outings. Any occasions of service that focussed on improving outdoor journeys were counted. A written summary of each team's performance was provided to teams within eight weeks by AM.

Feedback of results from the first audit was provided to each team about their compliance with key criteria, with comparison to the overall compliance by the five teams. Each team then set targets for the next 12 months (e.g., ' $50 \%$ of people with stroke will have written evidence that driving has been discussed').
A second retrospective audit of medical records was conducted 12 months later using identical tools and processes to the first audit. Medical files were requested of 100 people with stroke treated after the half-day implementation training workshop (20 consecutive records for each of the five teams). Nine rehabilitation professionals audited the medical records in addition to AM.

Barrier identification was conducted concurrently with the audit process. To identify barriers, we used two methods that have been recommended for implementation research [12]. First, we conducted in-depth interviews (described elsewhere [20]) with allied health professionals from two teams, and then transcribed and analysed the content. Interviewees were asked to describe what they knew about the outdoor journey intervention, including the published evidence, and factors that might help or hinder their team from implementing the outdoor journey intervention. Prompt questions were used to enquire about skills and knowledge, staffing, resources, assessment procedures, screening and report-writing systems, and treatment routines. Findings were then used to inform the content of a workshop.

\section{Education}

A half-day workshop was run in August 2007. The workshop was lead by AM. First, we presented a critical appraisal of the original randomised trial by Logan and colleagues [7], and a description of the complex outdoor journey intervention [10]. Therapists were alerted to the national clinical guideline recommendation about the intervention [11].

Second, baseline audit data were presented with the permission of the five teams. Based on the review by Grimshaw and colleagues [19], consensus was reached at the workshop that a $10 \%$ improvement in the target practice behaviours would be the goal for teams following the implementation program (i.e., the predetermined minimum clinically worthwhile difference).

Third, a written document was presented and discussed ('Increasing outdoor journeys after stroke: Protocols for use by rehabilitation professionals'). Protocols were provided for upgrading walking, bus and train travel training, trialling motorised scooters, addressing return to driving, and providing written information about transport options. These protocols had been prepared by the AM with advice from local team members.

Fourth, two case studies were presented by occupational therapists who had delivered escorted journeys to people with stroke. Each case study included goals of the person with stroke, treatment progression, and safety tips. A videotaped interview was also presented showing a person with stroke who described the benefits of being assisted to get out of the house. Participants 
then practiced writing sample goals related to outdoor journeys and community participation.

Finally, potential barriers and enablers to delivering the outdoor journeys were identified, then discussed by workshop participants in pairs or teams. Examples and quotes were presented from the earlier in-depth interviews conducted with team members [20]. Participants identified strengths, weaknesses, opportunities, and threats affecting their team's ability to provide the evidence-based outdoor journey intervention. Solutions were proposed, discussed and documented by team leaders.

\section{Outcome measures}

\section{Team outcomes}

The primary outcome of team behaviour change was the proportion of people with stroke who received six or more outdoor journey sessions from an occupational therapist, physiotherapist, or therapy assistant. These outcomes were obtained from the same medical record audits that were used to provide feedback to participating teams. Records were requested of consecutive people with stroke seen by teams for 12 months before (preintervention) and 12 months after the implementation (post-intervention) training workshop. Secondary outcomes, also obtained from medical record audits, included the proportion of people with stroke who were screened and asked questions about outings, their preferred destinations and modes of travel, and driving status.

\section{Patient outcomes}

Consecutive people with stroke from two teams who received the outdoor journey intervention and provided consent were visited at home by AM. They were visited on two occasions, once before therapy sessions commenced (baseline) and then again three months later (follow-up). Participants were asked a single question, which was the primary outcome of interest: 'Are you getting out of the house as often as you would like?' (yes/no). Four standardised measures were also completed with assistance from AM, partly to identify a suitable primary outcome measure for a future trial. First, participants completed the Nottingham Extended Activities of Daily Living (NEADL) scale [21], which is a selfreport measure comprising 22 questions about community and home-based activities (maximum score 66). The Life Space Assessment (LSA) [22] was also used; this self-report measure records how far a person has walked or travelled in the past month (maximum score 120). The Falls Efficacy Scale (International, FES-I) [23] enquired about concerns regarding the possibility of falling when performing, or thinking about performing, various activities (maximum score 64). The Reintegration to Normal Living Index (RNLI) [24] then measured how well a participant felt they had resumed communitybased activities (maximum score 22).

Finally, a list was generated of outings and outdoor journeys completed over the previous seven days, supervised or unsupervised, on foot or in a vehicle. An outing was defined as an excursion into the community beyond the front gate. An outdoor journey was defined as any excursion beyond the front or back door of the house, and included short walks to the post-box or around the garden. An excursion involving a walk to the car, then a car journey to the shops, then a walk into a shopping mall represented one outing but three outdoor journeys. This method of recording outings and outdoor journeys was replicated from the original trial by Logan and colleagues [7].

\section{Ethical approval}

Ethical approval for the study was obtained from the local area health service (Ref No. 2007/019) and university ethics committee (Ref No. 10092).

\section{Sample size}

While therapists agreed on a $10 \%$ improvement for the target practice behaviour $[18,19]$, the proportion of people with stroke who received six or more outdoor journey sessions, our study was not powered to detect this difference. This would have required recruitment of many more teams, and was beyond the scope of this pilot study that aimed to test the feasibility of the implementation program.

\section{Data analysis}

Team and patient outcome data were analysed using descriptive statistics including proportions, means/standard deviations, or median/interquartile range. For categorical data and proportions, we used McNemar's repeated measures chi-square test to compare withingroup differences. Mean within-group differences were calculated using paired $t$-tests and $95 \%$ confidence intervals for continuous data (NEADL, LSA, FES-I and HADS).

\section{Results}

\section{Sample characteristics}

\section{Rehabilitation team participant characteristics}

Of the 12 rehabilitation therapists who helped conduct the audits, all except one were female, and all were either an occupational therapist $(\mathrm{n}=8)$ or a physiotherapist $(\mathrm{n}=4)$.

\section{Patient participant characteristics}

For the pre-intervention cohort of people with stroke $(\mathrm{n}=77)$, the median age was 67.5 years (IQR 54.8 to 77.8 ); this cohort were a median of 23.5 days post-discharge from hospital or days since referral to the team (IQR 11.0 to 58.8). For the post-intervention cohort $(\mathrm{n}=53)$, the median age was 66.5 years (IQR 50.6 to 75.7); this cohort were a median of 21.5 days 
post-discharge from hospital or days since referral to the team (IQR 8.0 to 41.6 ).

\section{Medical record audit data}

Pre-intervention, 77 of the 100 medical records requested were available for auditing. A year later, when another 100 consecutive records were requested, we located and audited 53 medical records. Some medical records did not contain therapists' notes, while other records were not available for audit. Table 1 presents a summary of audit criteria and the proportion of medical records that complied with each criterion across teams.

At the 12-month audit, several notable changes in practice were recorded ( $\geq 10 \%$ change) including better recording and more frequent screening of people with stroke about their driving status $(+24 \%)$, noting of: preferred modes of travel $(+26 \%)$ and weekly outings $(+15 \%)$. The post-intervention audit also revealed better recording and more frequent delivery of outdoor journey sessions (19\% more people received one session; $15 \%$ more people received six sessions). A greater proportion of people with stroke $(76 \%)$ received at least one outdoor journey session compared to pre-intervention (57\%).

Audit data revealed a modest change in practice across teams, although this difference was not statistically significant. Nearly one-third of people with stroke $(32 \%)$ received six or more sessions after one year, compared to $17 \%$ at baseline (a 15\% change). However, there were marked differences between teams (see Table 2). Team four achieved the greatest change in practice (a $34 \%$ change). Initially, $36 \%$ of people with stroke whose records were audited received six or more
Table 2 Proportion of medical records audited where people with stroke received six or more outdoor journey sessions ( $n$ *, \%)

\begin{tabular}{|c|c|c|c|c|}
\hline \multirow[t]{3}{*}{ Team } & \multicolumn{4}{|c|}{ Time of Audit } \\
\hline & \multicolumn{2}{|c|}{$\begin{array}{l}\text { Pre-Intervention } \\
\text { (2006 to 2007) }\end{array}$} & \multicolumn{2}{|c|}{$\begin{array}{l}\text { Post-Intervention } \\
\text { (2007 to 2008) }\end{array}$} \\
\hline & $n *$ & $\%$ & $n *$ & $\%$ \\
\hline Team one & $4 / 22$ & 18.2 & $2 / 19$ & 10.5 \\
\hline Team two & $3 / 21$ & 14.3 & $6 / 15$ & 40.0 \\
\hline Team three & $2 / 13$ & 15.4 & $2 / 7$ & 28.6 \\
\hline Team four & $4 / 11$ & 36.4 & $7 / 10$ & 70.0 \\
\hline Team five & $0 / 10$ & 0.0 & $0 / 2$ & 0.0 \\
\hline Total & $13 / 77$ & 16.9 & $17 / 53$ & 32.1 \\
\hline
\end{tabular}

* ' $n$ ' refers to the number of audited files that contained evidence of outdoor journey sessions, divided by the total number of files audited per team

outdoor journey sessions. One year later, this proportion had increased to $70 \%$ for team four.

\section{Number of outdoor journey sessions}

The number of sessions per person increased from a mean of 2.2 (SD 3.2) at baseline to 4.5 (SD 7.9) after 12 months (median 1.0, IQR 0.0 to 3.0, to median of 2.0, IQR 0.0 to 7.0) (Figure 1). Team four successfully delivered a mean of 7.0 sessions (SD 4.3). Although team two increased the mean number of sessions, their follow-up data were skewed by one person with stroke who received 52 sessions. When that outlier was removed from analysis, the follow-up mean for that team decreased to 3.7 sessions (SD 4.3).

Patient outcomes

Outcome data were collected from 23 people with stroke who received outdoor journey sessions from two

Table 1 Audit data from medical records across five teams at baseline and follow-up 12 months later

\begin{tabular}{|c|c|c|c|c|c|}
\hline \multirow[t]{2}{*}{ Criteria } & \multicolumn{4}{|c|}{ \% Compliance } & \multirow[t]{2}{*}{$\%$ Change } \\
\hline & \multicolumn{2}{|c|}{$\begin{array}{l}\text { Baseline } \\
(\mathrm{N}=77)\end{array}$} & \multicolumn{2}{|c|}{$\begin{array}{l}\text { Follow-up } \\
(\mathrm{N}=53)\end{array}$} & \\
\hline Intervention: Is there written evidence of intervention aimed at increasing outdoor journeys & n & $\%$ & $n$ & $\%$ & \\
\hline Six sessions or more & 13 & $17 \%$ & 17 & $32 \%$ & $+15 \%$ \\
\hline Four sessions or more & 16 & $21 \%$ & 19 & $39 \%$ & $+18 \%$ \\
\hline Two sessions or more & 27 & $35 \%$ & 25 & $51 \%$ & $+16 \%$ \\
\hline At least one session & 44 & $57 \%$ & 37 & $76 \%$ & $-19 \%$ \\
\hline No sessions provided & 33 & $43 \%$ & 16 & $13 \%$ & $-30 \%$ \\
\hline Screening Questions: Were the following content areas documented? & $n$ & $\%$ & $n$ & $\%$ & \\
\hline Mobility status & 77 & $100 \%$ & 53 & $100 \%$ & $0.0 \%$ \\
\hline Home access & 69 & $90 \%$ & 47 & $89 \%$ & $-1.0 \%$ \\
\hline Pre-stroke driving status & 37 & $48 \%$ & 38 & $72 \%$ & $+24 \%$ \\
\hline Preferred destinations & 19 & $25 \%$ & 24 & $45 \%$ & $+20 \%$ \\
\hline Preferred modes of travel & 27 & $35 \%$ & 34 & $61 \%$ & $+26 \%$ \\
\hline Reasons for limited outings & 26 & $34 \%$ & 21 & $40 \%$ & $+6.0 \%$ \\
\hline Current outings discussed & 39 & $51 \%$ & 35 & $66 \%$ & $+15 \%$ \\
\hline Number of weekly outings estimated & 11 & $14 \%$ & 16 & $30 \%$ & $+16 \%$ \\
\hline
\end{tabular}

Medical records were audited across five community rehabilitation teams 


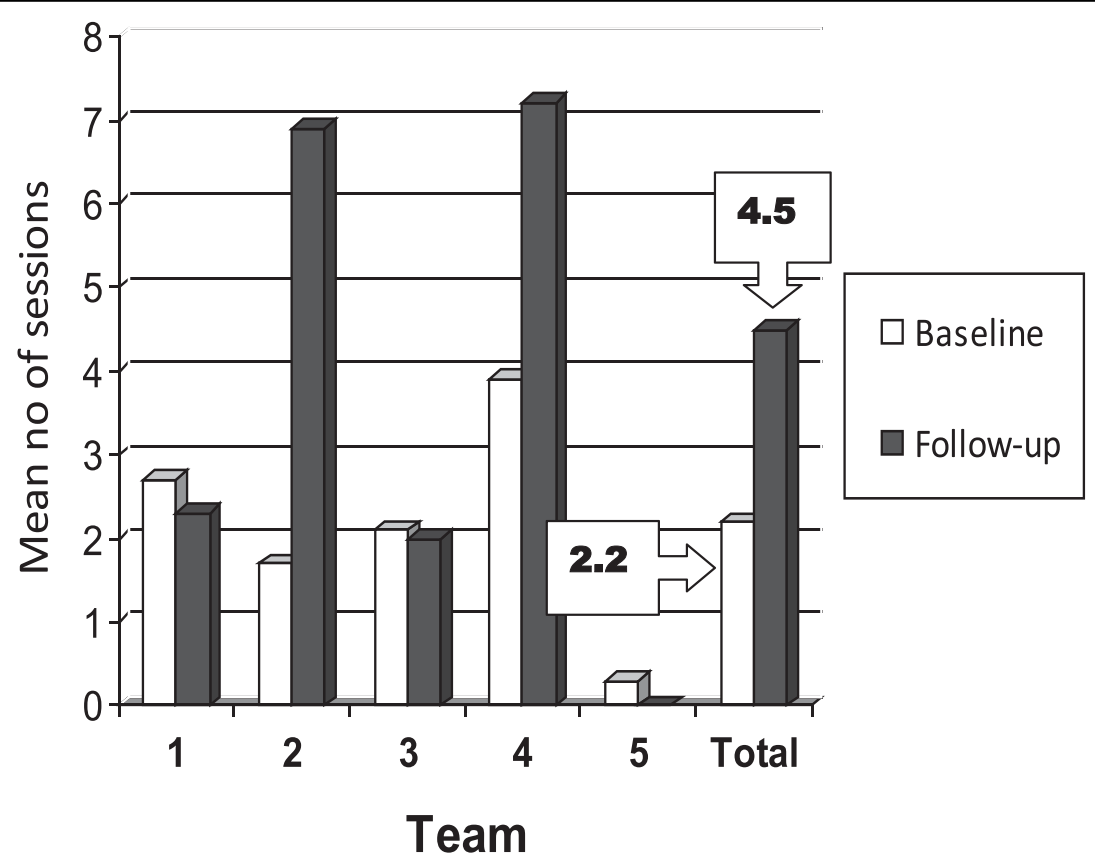

Figure 1 Mean number of outdoor journey sessions delivered by the five community teams as documented in medical records at baseline and follow-up

of the participating teams (see Table 3). The mean age of the sample was 66.7 (SD 12.8), one-half were female ( $\mathrm{n}=10,56.5 \%)$, and two-thirds drove a car pre-stroke ( $\mathrm{n}=15,65.2 \%)$. Median time to baseline data collection and commencement of the outdoor journey intervention was 58 days post-stroke (IQR 49 to 111), and 21 days post-discharge (IQR 7 to 40 ). Only one-third of the sample $(34.8 \%)$ said that they were getting out as often as they wanted before the outdoor journey sessions began.

When pre-post outcomes were calculated across this small sample, within-group differences only reached statistical significance for the NEADL (7.3 points, $95 \%$ CI, 1.2 to $13.5, \mathrm{p}=0.022)$ and FES-I (8.2 points, $95 \%$ $\mathrm{CI}, 2.0$ to $14.4, \mathrm{p}=0.012)$. For the key patient outcome of interest-the proportion of people with stroke who

Table 3 Within-group differences" after three months for people with stroke who received the outdoor journey intervention (Mean/SD) and provided pre-post data $(n=21)$

\begin{tabular}{|c|c|c|c|c|c|}
\hline Measure & Pre-test & Post-test & Diff & $95 \% \mathrm{Cl}$ & $P$ value \\
\hline 'Are you getting out of the house as often as you want?' (\% Yes) & $34.8 \%(n=8)$ & $57.1 \%(n=12)$ & $22.3 \%$ & NA & 0.219 \\
\hline Number of outdoor journeyst per week & $28.2(18.2)$ & $30.4(14.3)$ & 2.2 & -9.6 to 5.3 & 0.548 \\
\hline NEADL $(0-66)$ & $26.9(12.6)$ & $35.1(13.5)$ & 7.3 & 1.2 to 13.5 & $0.022 *$ \\
\hline RNLI (0-22) & $13.9(5.0)$ & $15.8(3.1)$ & 1.9 & -4.2 to 0.4 & 0.102 \\
\hline LSA $(0-120)$ & $36.4(13.8)$ & $40.7(15.2)$ & 4.3 & -12.9 to 4.4 & 0.314 \\
\hline FES-I (0-64) & $34.8(13.8)$ & $26.6(12.1)$ & 8.2 & 2.0 to 14.4 & $0.012 *$ \\
\hline HADS-A (0-21) & $5.7(4.8)$ & $6.0(4.5)$ & 0.2 & -2.1 to 1.6 & 0.766 \\
\hline HADS-D (0-21) & $5.5(4.7)$ & $6.6(3.8)$ & 0.7 & -2.2 to 0.7 & 0.271 \\
\hline Number of outings per week & $8.5(5.0)$ & $8.6(5.3)$ & 0.1 & & \\
\hline Number of days out the house: beyond the front door & $5.3(1.8)$ & $6.2(0.8)$ & 0.9 & & \\
\hline Number of days out the house: beyond the front gate & $4.3(2.1)$ & $4.2(2.2)$ & -0.1 & & \\
\hline
\end{tabular}

"Within-groups differences and confidence intervals calculated using paired t-tests (2-tailed), $\mathrm{n}=21$. Diff $=$ Difference. $95 \% \mathrm{Cl}=95 \%$ confidence interval. * Statistically significant at 0.05 .

NEADL = Nottingham Extended ADL index; RNLI = Reintegration to Normal Living Index; FES-1 = Falls Efficacy Scale International; HADS = Hospital Anxiety and Depression Scale. For all measures except the FES-I and HADS, an increased total score represents improved performance or health.

${ }^{\dagger}$ Outdoor journeys were calculated by adding each 'leg' completed during an outing. For example, a person who walked to the car, travelled in a car to the shops, walked from the car into shops was recorded as having completed three outdoor journeys. 
reported getting out of the house as often as they wanted-the within-group difference did not reach statistical significance $(p=0.219)$. The mean number of outings reported per week remained unchanged over time: 8.5 (SD 5.0) at baseline, and 8.6 (SD 5.3) at follow-up. Nor was there any significant change in the mean number of outdoor journeys or number of days out the house beyond the front door or front gate (Table 3).

\section{Consenting rate for stroke patients}

Almost one-half of all people with stroke referred over the 12 -month period (52\%) did not need or want outdoor journey sessions. These individuals did not have community participation goals, and were already getting out as often as they wanted. Further, of the $48 \%$ of stroke patients who received the outdoor journey sessions, $69 \%$ consented to provide outcome data and $31 \%$ declined.

\section{Discussion}

To our knowledge, this is the first knowledge translation study involving community stroke rehabilitation teams. Previous studies have reported on the performance of stroke unit teams using clinical audits in hospital settings in England [25], the Netherlands [26], and Australia [27]. Until completion of this study, less was known about how community teams performed when translating evidence from stroke trials into practice.

There are three key messages from our study discussed in depth below. First, it was feasible for community teams to provide multiple outdoor journey sessions as part of their usual practice. Second, the level of behaviour change varied across teams. Third, the outdoor journey sessions led to improved outcomes for people with stroke.

\section{The sample}

The teams appeared to be representative of non-inpatient rehabilitation stroke services in Sydney. While no database of services exists, a telephone survey was conducted informally by AM in early 2009 to any known community and outpatient service for adults with a stroke in Sydney. Results identified only two stroke-specific services in operation. Other services consisted of: three generic day hospitals/centres; at least 12 community-based transitional services for older adults recently discharged from hospital; fewer than 10 generic community-based services; and at least 15 hospital-based generic out-patient services. All of these service models were represented in our sample.

Professionals delivering the outdoor journey sessions were experienced occupational therapists and physiotherapists; all had at least five years clinical experience. Junior and recently graduated professionals are rarely employed in these positions, because of the complex caseload and clinical reasoning required.

People with stroke in both audit cohorts were similar in terms of median age (67.5 and 66.5 years respectively) and time post-discharge (median 23.5 days and 21.5 days, respectively). The median age of people with stroke in Australian hospitals is 76 years (IQR 65 to 83) [28], therefore, our audit cohorts were younger. They may have had fewer co-morbidities, however we did not record this information because of limited time. Unfortunately, we also did not record time post-stroke. In the trial by Logan and colleagues [7], people who received outdoor journeys sessions were approximately one year post-stroke, and lived at home. The 23 people in our sample had experienced their stroke more recently (they were approximately two months post-stroke), and had only been home for about three weeks.

\section{Feasibility and safety of the outdoor journey sessions}

An important finding from this study was that therapists were able to adapt their practice over the 12-month period. It was feasible for some teams to incorporate the extra sessions into their busy programs by sharing sessions across disciplines. Role expansion and sharing were the main strategies contributing to team behaviour change, as we have reported elsewhere [20]. In the trial by Logan and colleagues [7], only occupational therapists delivered the outdoor journey sessions. However, the sessions can be delivered by physiotherapists as well as occupational therapists (Dr Pip Logan, personal communication, November 2007). In our study, some sessions were also provided by a therapy assistant. We can recommend this strategy of role sharing to other teams in future studies.

No adverse events occurred, although professionals were concerned about risk management when escorting people out into the community. Stories collected from 19 of the 23 people with stroke will be used to inform future stroke participants of the process of getting out of the house with therapy support (Barnsley, McCluskey \& Middleton, What people say about travelling outdoors after a stroke: A qualitative study, submitted). Risk management strategies, such as health professionals' carrying a mobile phone and the number of a key family member, may help to alleviate concerns. People with stroke and their families can be assured that they will be well supervised, and their program upgraded safely and gradually.

Finally, it was feasible for two teams to recruit 23 people with stroke over 12 months, and consent them for outcome data collection. We had anticipated collecting data from 40 people with stroke (20 per team) in this time period, based on referrals from the previous year. However, participant numbers were about one-half of 
what we had anticipated. When we examined the data from one team, we found that less than $50 \%$ of their stroke caseload had outdoor mobility and community participation goals and wanted the outdoor journey intervention; of this sub-group, two thirds (69\%) were recruited and provided outcome data (33\% of their total stroke caseload). Therefore, about one-third of people with stroke referred to that service were eligible and consented. It is possible that team members engaged in gatekeeping, and did not recruit all eligible participants, as we observed in a previous feasibility study [29]. An independent recruiter may help to minimise this problem in future studies.

\section{Variations in the level of behaviour change and team functioning}

Team four out-performed other teams in the pre-intervention and post-intervention medical record audits; they had higher compliance with audit criteria, provided more outdoor journey sessions per patient, and (anecdotally) engaged in more role sharing. Yet Team four employed three different occupational therapists during the year. They did not have a stable team who had worked together for many years. Team and staffing changes were experienced by all but one of the teams during the 12-month study period. However, team four had a team leader who allocated time to quality improvement, systems change, and who orientated new therapists to the project during the year.

Team functioning and characteristics have been the focus of at least two large national studies to improve outcomes post-stroke in the Netherlands [26] and United States [30]. The Dutch study recruited 14 national stroke services, paying each $€ 15,000$ to cover program costs. Teams attended four conferences on service improvement, decided on problems and bottlenecks in their service, set goals, received coaching, support, and regular feedback on their performance, as well as site visits. Team characteristics and functioning explained $40 \%$ of the variance in hospital length of stay across services. It is possible that these domains explain differences in team outcomes in our study, but we cannot be sure because team functioning was not assessed.

In the North American study, the primary aim was to test whether team training enhanced team functioning and improved outcomes post-stroke [30,31]. Training for experimental teams included financial support ( $\$ 1,000$ per site), a 2.5 day workshop with follow-up meetings for team leaders [32] covering topics such as team problem solving, how to use program evaluation data, and write action plans. All teams received performance feedback. Stroke patients treated by experimental teams improved by 13.6 points more than control participants on the motor items of the Functional
Independence Measure. However, there was no statistically significant difference in the average length of hospital stay. Thus, there is a small but growing body of research suggesting that team coaching and training can enhance performance and improve patient outcomes. Future knowledge transfer studies should consider ways to measure, and strategies to enhance, team functioning.

\section{Fidelity of the intervention}

One factor that we tried to maximise in this study was fidelity of the original intervention. Implementation fidelity is the degree to which programs are implemented as intended by the original developers $[33,34]$. Unless an evaluation is made of fidelity, service providers cannot determine if a lack of impact is due to poor implementation or problems with the program itself [33]. We wanted to ensure that what local therapists were delivering was 'true' to Logan's original randomised trial and used a number of strategies to maximise fidelity. First, the first author spent time face-to-face in 2005 and 2008 with the trialist, Dr Pip Logan, discussing the intervention. Second, a 60-page protocol was developed and provided to professionals. No such document previously existed, and can form the basis of protocols for future studies. Third, we interviewed 19 people with stroke after their sessions had concluded and eight team members about their practice, in mid 2008, prior to the second audit. We did not, however, observe sessions, and cannot be sure that what therapists recorded in the medical records reflected what they did.

\section{Study limitations}

Our research had some limitations. First, the study was not powered to detect statistically significant differences in team or patient outcomes. We did however test the feasibility of multiple patient outcome measures to determine which instruments should be used in a future trial. Second, the absence of a control group and blinded assessor are major limitations. We do not know if the changes in team behaviour were due to the 'Out-andAbout Implementation Program' or factors related to the teams and health environment at the time. Our next study, a cluster randomised controlled trial, will address this limitation by randomising teams, include control teams that receive no audit feedback, no education and do not engage in the process of barrier identification.

\section{Implications for practice and research}

First, the current study highlights the complexity and challenges of changing practice behaviours. Small changes in practice, with large variations across teams can be expected with the first wave of implementation. Changes in the vicinity of $50 \%$ to $75 \%$ are unrealistic [18], and cannot be expected. 
Second, this study has implications for routine clinical practice and education. These professionals were asked to change their practice. In some instances, change was achieved through collaboration between physiotherapists and occupational therapists, and involvement of therapy assistants. Role sharing and expansion are examples of organisational interventions [35]. A more in-depth examination of how therapists can maximise their roles may be of benefit to improve delivery of outdoor sessions to people post-stroke. Further, a process analysis alongside our proposed cluster randomised trial, examining teamwork and leadership, would also be of interest.

\section{Summary}

Our 'Out and About Implementation Program' was feasible and safe. No adverse events were recorded when therapists delivered the outdoor journey sessions to community dwelling people with stroke. The practicalities of incorporating extra sessions into already busy work schedules can be a major impediment to practice change. Yet, multiple outdoor journey sessions were implemented by therapists; improved screening of people with stroke was conducted by team members about outings, preferred destinations, and driving. Such screening may help to raise therapists' awareness of community participation post-stroke. While $57 \%$ of people with stroke reported getting out and about as often as they liked after receiving the outdoor journey sessions, there is room for further improvement. Fidelity of the patient intervention needs to be monitored in future studies. A well-designed cluster randomised controlled trial is warranted to test the effectiveness of the implementation program and its active components: audit and feedback, barrier identification, and tailored education.

\section{Acknowledgements}

During this study, Annie McCluskey held a NHMRC-NICS-HCF Health and Medical Research Foundation Fellowship (2007-2009). The study was also supported by a project grant from the National Stroke Foundation. None of these organisations were involved in, or influenced data collection or analysis, writing up of the manuscript, or the decision to submit this manuscript.

\section{Author details \\ ${ }^{1}$ Community-Based Health Care Research Unit, Faculty of Health Sciences, The University of Sydney, New South Wales, Australia. ${ }^{2}$ Royal Rehabilitation Centre Sydney, New South Wales, Australia. ${ }^{3}$ Nursing Research Institute, St Vincent's and Mater Health Sydney and the Australian Catholic University, New South Wales, Australia. ${ }^{4}$ National Centre for Clinical Outcomes Research (NaCCOR), Nursing and Midwifery, The Australian Catholic University, Australia.}

\section{Authors' contributions}

The first author conceptualised and planned the study, collected and analysed the data, and drafted the manuscript. The second author advised on study design and writing of the manuscript. Both authors read and approved the final manuscript.

\section{Competing interests}

The authors declare that they have no competing interests.

Received: 20 November 2009 Accepted: 29 July 2010

Published: 29 July 2010

\section{References}

1. National Stroke Foundation: Clinical guidelines for acute stroke management. Melbourne, Australia: National Stroke Foundation 2007.

2. Hill $K$, Ellis P, Bernhardt J, Maggs $P$, Hull S: Balance and mobility outcomes for stroke patients: A comprehensive audit. Australian Journal of Physiotherapy 1997, 43:173-180.

3. Mackintosh SFH, Goldie P, Hill K: Falls incidence and factors associated with falling in older, community-dwelling, chronic stroke survivors ( $>1$ year after stroke) and matched controls. Ageing Clinical and Experimental Research 2005, 17:74-81.

4. Fisk G, Owsley C, Pulley L: Driving after stroke: Driving exposure, advice and evaluations. Archives of Physical Medicine \& Rehabilitation 1997, 78:1338-1345.

5. Turnbull M: Return to driving following stroke: Prevalence and associated factors [Unpublished masters thesis]. Research masters The University of Sydney, Faculty of Health Sciences 2007.

6. van de Port I, Wood-Dauphinee S, Lindeman E, Kwakkel G: Effects of exercise training programs on walking competency after stroke: A systematic review. American Journal of Physical Medicine \& Rehabilitation 2007, 86:935-951.

7. Logan PA, Gladman JRF, Avery A, Walker MF, Dyas J, Groom L: Randomised controlled trial of an occupational therapy intervention to increase outdoor mobility after stroke. British Medical Journal 2004, 329:1372-1377.

8. Ada L, Dean C, Hall J, Crompton S: A treadmill and overground walking program improves walking in persons residing in the community after stroke: A placebo-controlled, randomized trial. Archives of Physical Medicine \& Rehabilitation 2003, 84:1486-1491.

9. Lord S, McPherson K, McNaughton H, Rochester L, Weatherall M: How feasible is the attainment of community ambulation after stroke: A pilot randomized controlled trial to evaluate community-based physiotherapy in sub-acute stroke. Clinical Rehabilitation 2008, 22:215-225.

10. Logan PA, Walker MF, Gladman JRF: Description of an occupational therapy intervention aimed at improving outdoor mobility. British Journal of Occupational Therapy 2006, 69:2-6.

11. National Stroke Foundation: Clinical guidelines for stroke rehabilitation and recovery. Melbourne, Australia: National Stroke Foundation 2005.

12. National Institute for Health and Clinical Excellence: How to change practice: Understand, identify and overcome barriers to change. London: National Institute for Health and Clinical Excellence 2007.

13. National Institute of Clinical Studies: Identifying barriers to evidence uptake. Melbourne, Australia: National Institute of Clinical Studies 2006.

14. Grol R, Wensing M: Selection of strategies. Improving patient care: The implementation of change in clinical practice Edinburgh: Elsevier Butterworth HeinemannGrol R, Wensing M, Eccles M 2005.

15. McCluskey A: Implementing evidence into practice. Evidence-based practice across the health professions Edinburgh: Churchill LivingstoneHoffmann T, Bennett S, Del Mar C 2010, 318-339.

16. Farmer A, Legare F, Turcot K, Grimshaw J, Harvey J, McGowan J, Wolf F: Printed educational materials: Effects on professional practice and health care outcomes. Cochrane Database of Systematic Reviews 2008, CD004398.

17. Jamtvedt G, Young J, Kristoffersen D, O'Brien MA, Oxman AD: Audit and feedback: Effects on professional practice and health care outcomes. Cochrane Database of Systematic Reviews 2006, CD000259.

18. Grimshaw J, Eccles M, Thomas R, Maclennan G, Ramsay C, Fraser C, Vale L: Toward evidence-based quality improvement: Evidence (and its limitations) of the effectiveness of guideline dissemination and implementation strategies 1966-1998. Journal of General Internal Medicine 2006, 21:S14-20.

19. Grimshaw JM, Eccles MP, Matowe L, Shirran L, Wensing M, Dijkstra R, Donaldson C: Effectiveness and efficiency of guideline dissemination and implementation strategies. Health Technology Assessment 2004, 8:1-72.

20. McCluskey A, Middleton S: Delivering an evidence-based outdoor journey intervention to people with stroke: Barriers and enablers experienced by community rehabilitation teams. BMC Health Services Research 2010, 10:18. 
21. Nouri F, Lincoln NB: An extended activities of daily living scale for stroke patients. Clinical Rehabilitation 1987, 1:301-305.

22. Baker P, Bodner E, Allman R: Measuring life-space mobility in communitydwelling older adults. Journal of the American Medical Association 2003, 51:1610-1614

23. Yardley L, Beyer N, Hauer K, Kempen G, Piot-Ziegler C, Todd C: Development and initial validation of the Falls Efficacy ScaleInternational (FES-I). Age \& Ageing 2005, 34.

24. Wood-Dauphinee S, Opzoomer A, Williams J, Marchand B, Spitzer W: Assessment of global function: The Reintegration to Normal Living Index. Archives of Physical Medicine \& Rehabilitation 1987, 69:583-590.

25. Rudd AG, Hoffman A, Irwin P, Pearson M, Lowe D: Stroke units: Research and reality. Results from the National Audit of Stroke. Quality and Safety in Health Care 2005, 14:7-12.

26. Schouten LMT, Hulscher MEJL, Akkermans R, van Everdingen JJE, Grol R, Huijsman R: Factors that influence the stroke care team's effectiveness in reducing the length of hospital stay. Stroke 2008, 39:2515-2521.

27. Cadilhac DA, Pearce DC, Levi CR, Donnan GA, on behalf of the Greater Metropolitan Clinical Taskforce and New South Wales Stroke Services Coordinating Committee: Improvements in the quality of care and health outcomes with new stroke care units following implementation of a clinician-led, health system redesign programme in New South Wales, Australia. Quality and Safety in Health Care 2008, 17:329-333.

28. National Stroke Foundation: National Stroke Audit Clinical Report: Acute services. Melbourne, Australia: National Stroke Foundation 2007.

29. Lannin NA, Clemson L, McCluskey A, Lin CC, Cameron ID, Barras S: Feasibility and results of a randomized pilot study of pre-discharge occupational therapy home visits. BMC Health Services Research 2007, 7:42.

30. Strasser DC, Falconer JA, Stevens AB, Uomoto JM, Herrin J, Bowen SE, Burridge AB: Team training and stroke rehabilitation outcomes: A cluster randomized trial. Archives of Physical Medicine \& Rehabilitation 2008, 89:10-15.

31. Strasser DC, Falconer JA, Herrin JS, Bowen SE, Stevens AB, Uomoto JM: Team functioning and patient outcomes in stroke rehabilitation. Archives of Physical Medicine \& Rehabilitation 2005, 86:403-409.

32. Stevens AB, Strasser DC, Uomoto JM, Bowen SE, Falconer JA: Utility of treatment implementation methods in a clinical trial with rehabilitation teams. Journal of Rehabilitation Research and Development 2007, 44:537-546.

33. Carroll C, Patterson M, Wood S, Booth A, Rick J, Balain S: A conceptual framework for implementation fidelity. Implementation Science 2007, 2:40.

34. Dusenbury L, Brannigan R, Falco M, Hansen WB: A review of research on fidelity of implementation implications for drug abuse prevention in school settings. Health Education Research 2003, 18:237-256.

35. Wensing $M$, Wollersheim $H$, Grol R: Organizational interventions to implement improvements in patient care: A structured review of reviews. Implementation Science 2006, 1:2.

doi:10.1186/1748-5908-5-59

Cite this article as: McCluskey and Middleton: Increasing delivery of an outdoor journey intervention to people with stroke: A feasibility study involving five community rehabilitation teams. Implementation Science 2010 5:59.

\section{Submit your next manuscript to BioMed Central and take full advantage of:}

- Convenient online submission

- Thorough peer review

- No space constraints or color figure charges

- Immediate publication on acceptance

- Inclusion in PubMed, CAS, Scopus and Google Scholar

- Research which is freely available for redistribution

Submit your manuscript at www.biomedcentral.com/submit 